\title{
Sphingomyelin in Brain and Cognitive Development: Preliminary Data
}

\author{
Nora Schneider, ${ }^{1}$ D Jonas Hauser, ${ }^{1}$ Manuel Oliveira, ${ }^{1}$ Elise Cazaubon, ${ }^{1}$ Sara Colombo Mottaz, ${ }^{1}$ B Barry \\ V. O'Neill, ${ }^{1}$ Pascal Steiner, ${ }^{1}$ and Sean C. L. Deoni ${ }^{2,3}$
}

https://doi.org/10.1523/ENEURO.0421-18.2019

${ }^{1}$ Société des Produits Nestlé SA, Nestlé Research, 1000 Lausanne, Switzerland, ²Department of Pediatrics, Brown University, Providence, RI 02912, and ${ }^{3}$ Memorial Hospital of Rhode Island, Pawtucket, RI 02860

\begin{abstract}
Sphingomyelin (SM) supports brain myelination, a process closely associated with cognitive maturation. The presence of SM in breast milk suggests a role in infant nutrition; however, little is known about SM contribution to healthy cognitive development. We investigated the link between early life dietary SM, later cognitive development and myelination using an exploratory observational study of neurotypical children. SM levels were quantified in infant nutrition products fed in the first three months of life and associated with myelin content (brain MRI) as well as cognitive development (Mullen scales of early learning; MSEL). Higher levels of SM were significantly associated with higher rates of change in verbal development in the first two years of life $(r=0.65$, $p<0.001$ ), as well as, higher levels of myelin content at 12-24 months, delayed onset and/or more prolonged rates of myelination in different brain areas. Second, we explored mechanisms of action using in vitro models (Sprague Dawley rat pups). In vitro data showed SM treatment resulted in increased proliferation $[p=0.0133$ and $p=0.0434$ at 4 and $10 \mathrm{~d}$ in vitro (DIV)], maturation $(p=0.467$ at $4 \mathrm{~d}$ DIV) and differentiation $(p=0.0123$ and $p$ $=0.0369$ at 4 and $10 \mathrm{DIV}$ ) of oligodendrocyte precursor cells (OPCs), as well as increased axon myelination ( $p=$ 0.0005 at $32 \mathrm{DIV})$. These findings indicate an impact of dietary SM on cognitive development in healthy children, potentially modulated by oligodendrocytes and increased axon myelination. Future research should include randomized controlled trials to substantiate efficacy of SM for cognitive benefits together with preclinical studies examining SM bioavailability and brain uptake.
\end{abstract}

Key words: brain development; cognitive development; infants; myelination; oligodendrocytes; sphingomyelin

\section{Significance Statement}

The presences of sphingomyelin (SM) in breast milk suggests it has an important role in infant nutrition. Ways of measuring SM in milk are only recently developed, meaning little is known about its role in brain development. In this study, we used two approaches to explore this further. First, we investigated the link between early life SM obtained from the diet with later brain development and brain structure in a group of healthy children. Second, we examined the mechanism behind these effects using experimental models of brain development. Our findings in children indicate a positive impact of SM on brain development supported by experimental models. Future studies need to investigate further to build on the findings of our preliminary work.

\section{Introduction}

Sphingomyelin (SM) is the most abundant eukaryotic sphingolipid and one of the major constituents of cell

Received October 30, 2018; accepted July 5, 2019; First published July 19, 2019.

S.C.L.D. receives salary and research support from Société des Produits Nestlé membranes (Gault et al., 2010). It is enriched in the CNS (Babin et al., 1993) and particularly abundant in the myelin sheath that surrounds neuronal axons (Pierre, 1984). SM 
has been reported to play an important role in cell processes (Merrill and Sandhoff, 2002; Hirabayashi and Furuya, 2008), the regulation of inflammatory responses (Assi et al., 2013; Maceyka and Spiegel, 2014), and signal transduction (Lingwood and Simons, 2010). Moreover, due to its critical role in myelin integrity and function (Don et al., 2014) as well as in axonal maturation (Ledesma et al., 1999), SM may be a relevant lipid during brain development. In particular, SM may play a prominent role from mid-gestation to the end of the first postnatal year when CNS myelin dramatically increases (Tau and Peterson, 2010). Ensuring efficient transmission of nerve impulses along neuronal axons (Fields, 2008; Stiles and Jernigan, 2010), CNS myelination is important in the maturation of brain networks, coordinated information processing and ultimately cognitive performance in infants and children (O'Muircheartaigh et al., 2013, 2014; Deoni et al., 2014). Therefore, SM may play an important role in cognitive development via its structural and functional involvement in CNS myelination.

$\mathrm{SM}$ is present in human breast milk and is the major phospholipid in human milk fat accounting for $\sim 37 \%$ of the phospholipid fraction (Bitman et al., 1984; Oshida et al., 2003). Mature breast milk contains SM at levels between 31 and $153 \mathrm{mg} / \mathrm{l}$ (Cilla et al., 2016), indicating high variability. However, despite these levels in breast milk, the functional role of dietary SM on cognitive development or its physiologic impact on underlying neurodevelopmental processes like myelin formation, remains poorly understood. In fact, only one dietary intervention study has evaluated the effect of SM supplementation on cognition in human infants. In a sample of low-birth weight infants, the feeding of SM-fortified infant formula ( $20 \%$ vs $13 \%$ total milk phospholipids) in the first eight weeks of life resulted in increased plasma and erythrocyte SM levels at four, six, and eight weeks of life. In addition, the same study reported improved behavior rating scores (behavior rating scale of the Bayley scales of infant and toddler development; BSID-II), better novelty preference scores (Fagan test), lower latency of visual evoked potentials and increased sustained attention scores (free-play sustained attention test of Colombo) at 12 and/or 18 months of age; no differences were found for neurodevelopment scores (BSID-II; Tanaka et al., 2013). The underlying mechanism for those findings were not investigated, but improvements in myelination (potentially influenced by $\mathrm{SM}$ ) were speculated. In another randomized controlled

designed research; N.S., J.H., M.O., and S.C.L.D. performed research; N.S., J.H., M.O., E.C., and S.C.L.D. analyzed data; N.S., J.H., M.O., E.C., S.C.M., B.V.O., P.S., and S.C.L.D. wrote the paper.

The in vitro work was funded by Société des Produits Nestlé SA. The observational cohort study was supported by the National Institutes of Mental Health Grant R01 MH087510 and the Bill and Melinda Gates Foundation Grant OPP1120016.

Correspondence should be addressed to Barry V. O'Neill at barryvincent.oneill@rd.nestle.com.

https://doi.org/10.1523/ENEURO.0421-18.2019

Copyright (C) 2019 Schneider et al.

This is an open-access article distributed under the terms of the Creative Commons Attribution 4.0 International license, which permits unrestricted use, distribution and reproduction in any medium provided that the original work is properly attributed. trial in healthy infants a low-energy, low-protein formula supplemented with bovine milk fat globule membranes, containing SM, was compared to a standard formula and to a reference group of breastfed infants. The intervention (two to six months of life) resulted in higher cognitive scores at 12 months of age in the experimental group versus control group. It also resulted in similar cognitive scores when comparing the experimental group to the breastfed infants (Timby et al., 2014). These studies suggest SM is a relevant nutritional contributor to brain and cognitive development.

Alongside the aforementioned clinical investigations, previous rodent studies provide support for SM in brain and cognitive development. Specifically, a SM-enriched diet positively affects CNS myelination and myelin thickness in experimental conditions of low serine palmitoyltransferase (SPT) activity, a key enzyme for the biosynthesis of sphingosine and a precursor of many sphingolipids, including SM (Oshida et al., 2003). With regard to cognitive performance, early life rats supplemented with a $1 \%$ complex milk lipid, which includes SM, resulted in an improved novelty memory performance and an improvement in learning of a spatial memory task in adulthood (Vickers et al., 2009).

In summary, the enriched presence of SM in the CNS and maternal breast milk, alongside its involvement in myelination suggest a prominent role in early life brain and cognitive development. However, the impact of dietary intake of SM as well as underlying mechanisms of action are mostly unknown, especially in healthy infants and young children. We investigated the link between early life dietary SM, developmental myelination and later cognitive development in a cohort of neurotypical infants and young children. Furthermore, we investigated potential mechanisms for SM-related effects on myelination using in vitro models.

\section{Materials and Methods}

\section{Observational cohort study}

Infants and children recruited for this study were recruited from the Brown University Assessment of Myelination and Behavior Across Maturation (BAMBAM) as part of an accelerated-longitudinal study focused on characterizing normal brain development. Specific exclusion criteria included in utero alcohol or illicit substance exposure, premature ( $<37$ weeks of gestation) or multiple birth, fetal ultrasound abnormalities, complicated pregnancy (e.g., preeclampsia), Activity, Pulse, Grimace, Appearance, Respiration (APGAR) scores $<8$, neonatal intensive care unit admission, neurologic disorder (e.g., head injury, epilepsy), psychiatric or developmental disorders in the infant, parents or siblings (including maternal depression requiring medication). Eligibility was determined via a pre-enrolment interview and verified by family and medical history questionnaire updates throughout the study. To date, $>450$ typically developing infants and toddlers have been recruited and assessed at regular intervals through infancy and childhood. Infants $(N=236$, 132 male), defined as children under two years of age, have received biannual MRI and neurocognitive assess- 
Table 1. Demographic information

\begin{tabular}{|c|c|c|c|c|c|c|c|c|c|c|c|}
\hline Gender & $\begin{array}{c}\text { Male }(n) \\
\text { Female }(n)\end{array}$ & $\begin{array}{r}\text { All inf } \\
54 \\
34\end{array}$ & ants & $\begin{array}{r}\text { Prodi } \\
2 \\
1\end{array}$ & ct A & Produ & ict B & $\begin{array}{r}\text { Produ } \\
12 \\
9\end{array}$ & ct C & ANOVA $p$ & $\chi^{2} p$ \\
\hline Marital status & Married/living together $(n)$ & 68 & & 29 & & 21 & & 18 & & & 0.57 \\
\hline Age range $(d)$ & & $88-340$ & & $88-33$ & & $98-33$ & & $103-3$ & 409 & & \\
\hline & & Mean & SD & Mean & SD & Mean & SD & Mean & SD & & \\
\hline Gestation (d) & & 281 & 19 & 285 & 12 & 275 & 11 & 278 & 6 & $A$ vs $B, p<0.01$ & \\
\hline Number of scans per child & & 2.5 & 1.3 & 2.1 & 1.3 & 3.0 & 1.2 & 2.2 & 1.4 & $A$ vs $B, p=0.01$ & \\
\hline Mean inter-scan interval (d) & & 380 & 250 & 330 & 190 & 407 & 299 & 439 & 250 & 0.21 & \\
\hline
\end{tabular}

ments from age at enrolment until two years of age, with annual assessments thereafter. Toddlers ( $N=218,122$ male), defined as children older than two years, have received annual $\mathrm{MRI}$ and neurocognitive assessments from age of enrolment. Though an accelerated-longitudinal study design, some children have to-date received only one scan and assessment. Thus, there is a combination of cross-sectional and longitudinal data available. This study was conducted according to the guidelines laid down in the Declaration of Helsinki and all procedures involving human subjects were approved by the host institution ethical committee [Brown University, 0911000083]. Written informed consent was obtained from all subjects.

For our exploratory and retrospective observational study on dietary SM, brain and cognitive development, we selected all children from the larger study cohort with data on their early life feeding (i.e., exclusive infant nutrition product feeding). This resulted in $N=150$ infants and toddlers that could be considered for the specific analyses on the influence of SM intake on brain myelination and cognitive development. This group was further refined by including only those who could be grouped into the biggest homogenous infant nutrition product groups as available from the cohort, see further details in the next section.

\section{Nutritional intake and infant nutrition product composition analysis}

Infant nutrition product intake was provided by parents as part of child history information. For our study, only children receiving the same product for at least $80 \%$ of feeds during the first three months (90 d) of life were included. Three products were found to be predominant $(N=88)$, with $44 \%$ of children fed product $A, 32 \%$ fed product B, and $24 \%$ fed product C. Quantification of SM in products $A, B$ and $C$ was determined retrospectively at Neotron analytical laboratories (Italy) using the method of Giuffrida et al. (2013). This validated method has a SM quantification limit of $<200 \mathrm{mg} / \mathrm{kg}$. The products for analyses were obtained from commercial providers (local supermarket in the Rhode Island area) at the study end, not during the actual feeding period of the infants. Demographic information for each group is provided in Table 1. Groups were, in general, matched for gender composition, parent marital status, birth weight, maternal education (as denoted by the education scale of the Hollingshead Four-factor Index of socioeconomic status).
There was a significant group difference in gestational age between the Product A ( $\sim 40$ weeks) and product B children ( $\sim 39.3$ weeks); however, both groups are considered full term.

\section{Cognitive development}

To assess emerging cognitive abilities, all children underwent a neuropsychological assessment by trained and qualified medical and psychological staff within $7 \mathrm{~d}$ of a successful MRI. For the age group included herein, the cognitive outcome measure was the Mullen scales of early learning (MSEL; Mullen, 1995). The MSEL is a standardized and population-normed tool for measuring early cognitive abilities in children under 68 months of age through tasks of visual reception, expressive and receptive language, fine and gross motor function. In this study, we considered the verbal developmental quotient (VDQ), based on the receptive and expressive language subscales, the non-VDQ (NVDQ) based on the visual reception and fine motor subscales, as well as the early learning composite (ELC) based on the subscales visual reception, fine motor, expressive language, and receptive language. The composite scores can be calculated with a population mean of 100 and a SD of 15 .

\section{MR imaging}

MRI scans from infants were acquired during natural non-sedated sleep in a 3T Siemens Tim Trio scanner. To minimize motion, children were swaddled with a pediatric MedVac vacuum immobilization bag (CFI Medical Solutions) and secured with foam cushions. Scanner noise was reduced by reducing the imaging speed and using a noise-insulating scanner bore insert (Quiet Barrier HD Composite, Ultra Barrier). Pediatric ear covers and electrodynamic headphones (MR Confon) were also used (Dean et al., 2014a). For MRI sequences please refer to Table 2.

To assess brain myelin content and growth, neuroimaging with multicomponent-driven equilibrium single pulse observation of T1 and T2 (mcDESPOT; Deoni et al., 2008) was used to quantify the myelin water fraction (MWF), a surrogate measure of myelin volume, throughout the brain. Infants were scanned using optimized protocols described previously (Deoni et al., 2012) and detailed in Table 3. Total imaging times ranged from 18 to $25 \mathrm{~min}$, depending on the age of the child. 
Table 2. MRI sequences and parameters

\begin{tabular}{lcc}
\hline MRI sequence & Sequence parameters & Time \\
mcDESPOT & Age-group protocols drawn from Dean et al. $(2014 \mathrm{~b})$ & $18-25: 00$ \\
T1W MP-RAGE & $(1.2 \times 1.2 \times 1.2) \mathrm{mm}^{3}, \mathrm{TR}=1800 \mathrm{~ms}, \mathrm{TE}=2.2 \mathrm{~ms}, \mathrm{TI}=900 \mathrm{~ms}$, \\
& flip angle $=15^{\circ}, \mathrm{FOV}=220 \times 220 \mathrm{~mm}^{2}, 134$ slices, GRAPPA $=2$
\end{tabular}

MC DESPOT = multicomponent-driven equilibrium single pulse observation of T1 and T2; MP-RAGE = magnetization-prepared rapid gradient-echo; DTI = diffusion-tensor imaging; rSMRI = resting state MRI; TR = repetition time; TE = echo time; $\mathrm{TI}=$ inversion time; FOV = field of view.

Following data collection and standard data preprocessing (Deoni 2011), voxel-wise MWF maps were calculated (Deoni and Kolind, 2015) for each child and then non-linearly aligned to approximate Montreal Neurologic Institute (MNI) space (Avants et al., 2011; Deoni et al., 2012). White matter (WM) masks of five bilateral regions (frontal, temporal, occipital, parietal, and cerebellar WM) as well as the body, genu, and splenium of the corpus, were created from common WM atlases and superimposed onto each child's MWF map to derive mean MWF values for each region for each child.

\section{SM, brain myelin, and cognition}

For statistical analyses and a better understanding of developmental changes with age, we considered the following three groups based on age, and performed both cross-sectional and longitudinal assessments: (1) crosssectional analysis of children between 1 and 12 months of age ( $N=39,17$ male); 2$)$ cross-sectional analysis of children between 12 and 24 months of age $(N=21,8$ male); 3) longitudinal analysis of children between one month and six years of age ( $N=88,54$ male).

\section{Cross-sectional analysis}

For groups 1 and 2, the correlation between MWF and product SM content was evaluated at each image voxel throughout the brain using a simple linear correlation approach that accounted for child age. A general linear model (GLM) was constructed that modeled MWF as the outcome variable and product SM content and child age at time of scan as predictor variables. Analysis was performed at each image voxel with significance defined as $p$ $<0.05$ corrected for multiple comparisons using a threshold-free cluster-based technique (Smith and Nichols, 2009).

To compare measures of cognitive ability, an unpaired $t$ test was used to test the difference in ELC, VDQ, and NVDQ between children fed product $A$ and product $B$. The product $C$ group was omitted from this group analysis due to its small size in each age group. It was included in all other analyses. Significance was defined as $p<0.017(p<0.05$ corrected for the three analyses using Bonferroni correction).

\section{Longitudinal analysis}

Non-linear mixed-effects modeling was used to fit a modified Gompertz growth model (Dean et al., 2014b) to each child's longitudinally-acquired MWF data in each of the 13 WM regions of interest. The Gompertz curve is a sigmoidal function defined by four parameters: the initial onset of growth $(\beta)$, initial growth rate $(\gamma)$, a second reflection where growth begins to slow considerably $(\alpha)$, and a final linear growth rate $(\delta)$. Following estimation of these parameters for each individual, the correlation between each parameter and product SM content was calculated. Statistical significance was defined as $p<0.001$ ( $p<$ 0.05 corrected for the 52 tests).

Correlations were also investigated between product SM content and cognitive development trends in a similar manner. Linear mixed-effects modeling was first used to fit a linear model to each child's repeated ELC, VDQ, and NVDQ data. The correlation between SM content and the slope of the linear model was then calculated, with statistical significance defined as $p<0.017$ ( $p<0.05$ corrected for the three analyses using Bonferroni correction).

\section{Preclinical materials and methods \\ Cell treatments}

In vitro studies were performed using either pure oligodendrocyte precursor cell (OPC) cultures or co-cultures of OPC and primary neurons. Pure OPC and co-cultures were treated with either olesoxime at $0.3 \mu \mathrm{M}$, SM (liposomes composed of 75\% 1,2-dipalmitoyl-sn-glycero-3phosphocholine and 25\% bovine brain SM, Avanti Polar Lipids) at $0.5,5$, and $25 \mu \mathrm{M}$ or vehicle (1,2-dipalmitoylsn-glycero-3-phosphocholine liposomes). SM doses were selected to represent human CSF levels (Mandal et al., 2012) or higher. Olesoxime was used as positive control (Magalon et al., 2012). For pure OPC and coculture tests

Table 3. Details of the mcDESPOT acquisition protocol per age-group ${ }^{(25)}$

\begin{tabular}{llllc}
\hline \multicolumn{1}{c}{ Age group (months) } & \multicolumn{1}{c}{9} & \multicolumn{1}{c}{ 9-16-28 } \\
Acquisition time (min:s) $18: 22$ & \multicolumn{1}{c}{$3-9$} & $18: 42$ & $21: 38$ & $24: 20$ \\
Field of view $\left(\mathrm{cm}^{3}\right)$ & $14 \times 14 \times 13$ & $17 \times 17 \times 14.4$ & $18 \times 18 \times 15$ & $20 \times 20 \times 15$ \\
Unprotected dBa & 54 & 62 & 69 & 74 \\
SPGR TR/TE (ms) & $12 / 5.8$ & $12 / 5.9$ & $12 / 5.4$ & $11 / 5.2$ \\
SPGR flip angles $\left(^{\circ}\right)$ & $2,3,4,5,7,9,11,14$ & $2,3,4,5,7,9,11,14$ & $2,3,4,5,7,9,11,14$ & $2,3,4,5,7,9,12,16$ \\
IR-SPGR TI (ms) & $600 / 950$ & $600 / 900$ & $500 / 850$ & $500 / 800$ \\
bSSFP TR/TE (ms) & $10 / 5$ & $10.2 / 5.1$ & $10 / 5$ & $9.8 / 4.4$ \\
bSSFP flip angles $\left(^{\circ}\right)$ & $9,14,20,27,34,41,56,709,14,20,27,34,41,56,709,14,20,27,34,41,56,709,14,20,27,34,41,56,70$
\end{tabular}

SPGR = Spoiled gradient-echo; IR-SPGR = inversion recovery SPGR; TR = repetition time; TE = echo time; TI = inversion time; SSFP = steady-state free precession. 
Table 4. Mean cognitive scores per infant age and product group

\begin{tabular}{|c|c|c|c|c|c|c|c|c|}
\hline \multirow[t]{2}{*}{ MSEL scores } & \multicolumn{4}{|c|}{$\begin{array}{l}\text { 1-12 months } \\
M(S D)\end{array}$} & \multicolumn{4}{|c|}{$\begin{array}{l}\text { 12-24 months } \\
\text { M (SD) }\end{array}$} \\
\hline & All products & Product A & Product B & Product C & All products & Product $A$ & Product B & Product C \\
\hline ELC & $93.2(14.4)$ & $91.5(15.7)$ & 94.7 (13.8) & $94.3(15.2)$ & 97.1 (13.6) & $92.3(12.4)$ & 104.2 (8.5) & 101.8 (20.3) \\
\hline VDQ & $108.1(21.8)$ & $109.9(21.4)$ & $105.1(18.1)$ & $107.2(19.3)$ & $104.3(15.6)$ & 96.9 (12.1) & 109.5 (18.7) & 107 (18.8) \\
\hline NVDQ & $90.8(21.6)$ & $91.1(23.8)$ & $90.4(18.4)$ & 90.7 (16.3) & $101(17.8)$ & 98.8 (14.7) & $108.2(8.4)$ & 95 (13.2) \\
\hline
\end{tabular}

both were run in triplicates, for each replication the average of at least two technical replicates per condition per time point was calculated.

Pure OPC cultures

Cells were imaged at 4,7 , and $10 \mathrm{~d}$ in vitro (DIV). OPC proliferation, differentiation and maturation was determined by the total number of $4^{\prime}, 6$-diamidino-2phenylindole $(\mathrm{DAPI})+, \mathrm{O} 4+$, and myelin basic protein $(\mathrm{MBP})+$ cells, respectively.

Co-cultures

Ten DIV OPC were plated with 14 DIV neurons. Cocultures were fixed at 18, 26, and 32 DIV and stained for MBP and $\beta$-III-tubulin (marker of axonal growth); coexpression was used as an index of myelination.

For both pure OPC and co-cultures, the nutrient treatment started at 3 DIV (for coculture the DIV count started the day of the neuron-OPC coculture) and was administered via medium change every second day.

\section{OPC culture}

Cells from Sprague Dawley rat pup (P2) cortices were isolated using papain solution at $37^{\circ} \mathrm{C}$ for $20 \mathrm{~min}$, processed through a $100-\mu \mathrm{m}$ gasket and plated at 30,000 cells $/ \mathrm{cm}^{2}$ in neuronal medium (with $1 \%$ FBS) for $3 \mathrm{~d}$. On day 9 , a $45-$ min shaking (50 rpm) in $5 \% \mathrm{CO}_{2}$ was used to isolate OPC that were then kept for $3 \mathrm{~h}$ on $4 \mathrm{ml}$ of glial culture media (DMEM added with FBS 10\%, $33 \mathrm{U} / \mathrm{ml}$ penicillin and $33 \mu \mathrm{g} / \mathrm{ml}$ streptomycin, GlutaMAX 1\%) supplemented with $5 \mu \mathrm{g} / \mathrm{ml}$ insulin. Flask were shaken $16 \mathrm{~h}$ at $220 \mathrm{rpm}$ and OPC were isolated by pre-plating on a Petri dish for $30 \mathrm{~min}$, after which supernatant (containing the OPC) was collected. For OPC maturation, OPC were seeded at 30,000 cells $/ \mathrm{cm}^{2}$.

For OPC-neuron coculture myelination, collected OPC were seeded at 30,000 cells $/ \mathrm{cm}^{2}$ on hippocampal neurons in a final volume of $1 \mathrm{ml}$ oligodendrocyte media [OL media; DMEM added with $100 \times$ OL-Supplement, bovine insulin (from $1 \mathrm{mg} / \mathrm{ml}$ stock), GlutaMAX, Holo-transferrin (from 33 $\mathrm{mg} / \mathrm{ml}$ stock), B27 supplement, FBS, with CNTF (from 50 $\mathrm{ng} / \mu \mathrm{l}$ stock) added on subsequent medium changes].

\section{Primary neuronal cultures}

Hippocampus (E18 Sprague Dawley embryo) was incubated with $2.5 \%$ trypsin for $15 \mathrm{~min}$ at $37^{\circ} \mathrm{C}$, then gently washed and kept in culturing media. Following mechanical dissociation, cells were plated at $50,000 / \mathrm{cm}^{2}$ in neuronal plating medium and after $4 \mathrm{~h}$ put in compete neuronal culturing medium.

\section{Immunofluorescence quantification}

Images were taken with ArrayScan XTI HCA Reader (Thermo Fisher Scientific) equipped with a Photometrics $\mathrm{X} 1,14$ bit, high-resolution camera. A Zeiss Plan-
NEOFLUAR $20 \times / 0.4$ objective was used to capture 20 images per well (resolution of $1104 \times 1104$ pixels). Florescence was detected with the following settings: excitation LED 386_23 (DAPI), 549_15 (MBP) and 458_20 nm (O4 and $\beta$-III-tubulin), penta-band BGRFRN dichroic mirror, BGRFRN emission filter, exposure time of $0.0263 \mathrm{~s}$ (DAPI), $0.0553 \mathrm{~s}(\mathrm{MBP})$, and $0.266 \mathrm{~s}(\mathrm{O} 4)$ for pure OPC and $0.0233 \mathrm{~s}$ (DAPI), $0.1203 \mathrm{~s}$ (MBP), and $0.045 \mathrm{~s}(\beta-\mathrm{III}$ tubulin) for co-cultures, respectively. Image analysis was done with HCS Studio software (Thermo Fisher Scientific). MBP and $\beta$-III-tubulin colocalization fluorescence was quantified using $\beta$-III-tubulin area signal as a mask to determine regions of interest, then measuring the MBP signal in this region (threshold: signal $4 \times$ higher than the MBP background signal).

\section{Data analysis}

The effect of different doses of SM on the independent outcomes was assessed using a linear mixed model with treatment as fixed effect and the round as random effect. The effect size was represented by mean difference between groups, estimated by restricted maximum likelihood approach (REML). Data were a-priori divided by the average of the untreated group for each time point. Applying the Benjamini-Hochberg procedure to control the false positive discovery rate at $5 \%$ (on 84 performed tests), the $38 p<0.022$ can all be considered significant (Benjamini and Hochberg, 1995). All the results were expressed as mean (SEM) estimated by the model and the differences are considered to be statistically significant at $p<0.05$. Statistical analyses were performed using R.3.2.1.

\section{Results}

For statistical tables of each analyses, please see Table 6 and 7.

\section{Observational cohort study}

Nutritional composition analyses

SM levels ranged from 28 to $71 \mathrm{mg} / \mathrm{l}$ in the three analyzed infant nutrition products, with $28 \mathrm{mg} / \mathrm{l}$ in product A, $71 \mathrm{mg} / \mathrm{l}$ in product $\mathrm{B}, 28 \mathrm{mg} / \mathrm{l}$ in product $\mathrm{C}$.

\section{Cross-sectional results}

For an overview of the mean cognitive development scores, please refer to Table 4.

From the cross-sectional data spanning 1-12 months, we found no significant group difference in ELC $(p=$ $0.56), \operatorname{NVDQ}(p=0.56)$, or VDQ $(p=0.94)$. For the $12-$ to 24-month data, significant differences before correction were found for ELC $(p=0.02)$ and a trend toward significance for NVDQ $(p=0.09)$ and VDQ $(p=0.06)$, however, these did not remain statistically significant after correc- 


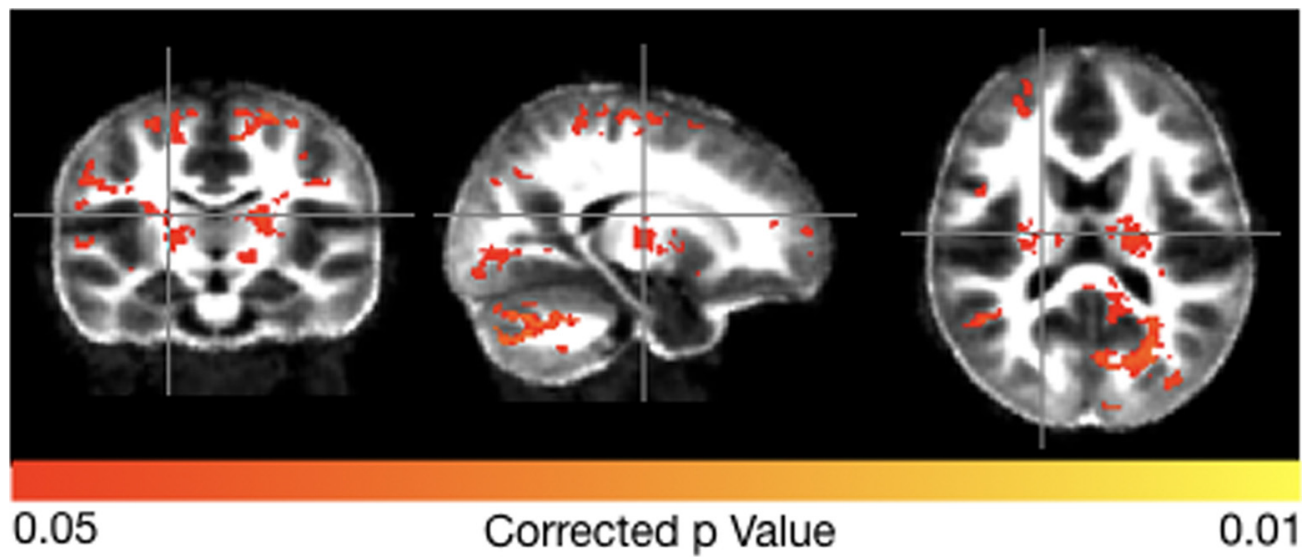

Figure 1. Brain regions with a significant relationship between product SM content and brain MWF in children 12-24 months of age. Colored brain regions are those where associations between SM content and MWF content reached statistical significance $(p<0.05$ FDR).

tion for multiple comparisons. With respect to myelin content, no significant correlations between MWF and product SM content was observed in the 1- to 12-month data; however, significant associations, corrected for multiple comparisons, were found in the 12- to 24-month data (Fig. 1) in bilateral cerebellum $(r=0.56, p=0.001)$, occipital lobe $(r=0.48, p=0.03)$, visual cortex $(r=0.44$, $p=0.04)$, internal capsule $(r=0.58, p=0.001)$, and parietal lobe and motor cortices $(r=0.52, p=0.02)$.

\section{Longitudinal results}

With respect to cognitive development, SM content was significantly and positively associated with the rate of change in VDQ (Pearson's $r=0.65, p<0.001$ ). No significant associations were found for change in NVDQ (Pearson's $r=0.09, p=0.52$ ) and overall ELC following multiple testing correction (Pearson's $r=0.28, p=0.06$ ). A summary of the longitudinal MWF growth for different brain regions and SM content analysis is shown in Table 5. Overall, we note that early life SM content is significantly related to brain myelin development profiles throughout the brain (Fig. 2; Table 5) and, in particular with respect to the initial onset of myelination (Gompertz curve parameter $\beta$ ), i.e., greater SM content being associated with a later myelin onset, and initial growth rate (Gompertz curve parameter $\gamma$ ), i.e., greater SM content being associated with a slower but more prolonged rate of myelination.

\section{In vitro study}

Results obtained from healthy infant observational study shows that dietary SM intake are correlated with increased level of myelination, suggesting that SM may affect the cellular mechanisms linked to myelin formation. to investigate how SM affects myelination, we first investigated how SM affects OPC proliferation, differentiation and maturation in pure OPC cultures, by measuring the total number of cells, $\mathrm{O} 4$ positive and MBP positive cells across time, Second, we characterized the effect of SM on axon myelination and axonal growth in an OPC and neurons coculture model by quantifying MBP and $\beta$-tubulin III signals alone or their respective colocalization for myelination (for more details, see Materials and Methods).

\section{Pure OPC cultures}

We observed $0.5 \mu \mathrm{M}$ SM resulted in increased total cell number, significantly at 4 DIV (effect size: $29.6 \%, p=$ 0.0133; Fig. 3A). SM at higher doses (5 and $25 \mu \mathrm{M}$ ) had no

Table 5. Correlation analysis of each Gompertz growth model and SM content

\begin{tabular}{|c|c|c|c|}
\hline Region & Parameter & Pearson's $r$ & $p$ value \\
\hline \multirow[t]{4}{*}{ Frontal WM } & $\alpha$ & 0.08 & 0.580 \\
\hline & $\beta$ & -0.51 & $<0.001 * * *$ \\
\hline & $\gamma$ & -0.21 & 0.147 \\
\hline & $\delta$ & -0.22 & 0.128 \\
\hline \multirow[t]{4}{*}{ Temporal WM } & $\alpha$ & 0.04 & 0.790 \\
\hline & $\beta$ & -0.99 & $<0.001 * * *$ \\
\hline & $\gamma$ & -0.83 & $<0.001 * * *$ \\
\hline & $\delta$ & 0.29 & $0.040 *$ \\
\hline \multirow[t]{4}{*}{ Parietal WM } & $\alpha$ & 0.37 & $0.008 * *$ \\
\hline & $\beta$ & -0.90 & $<0.001 * * *$ \\
\hline & $\gamma$ & -0.99 & $<0.001 * * *$ \\
\hline & $\delta$ & -0.97 & $<0.001 * * *$ \\
\hline \multirow[t]{4}{*}{ Occipital WM } & $\alpha$ & 0.13 & 0.170 \\
\hline & $\beta$ & -0.99 & $<0.001 * * *$ \\
\hline & $\gamma$ & -0.45 & $0.001 * * *$ \\
\hline & $\delta$ & 0.36 & $0.012 *$ \\
\hline \multirow[t]{4}{*}{ Cerebellar WM } & $\alpha$ & 0.99 & $<0.001 * * *$ \\
\hline & $\beta$ & -0.68 & $<0.001 * * *$ \\
\hline & $\gamma$ & -0.83 & $<0.001 * * *$ \\
\hline & $\delta$ & 0.31 & $0.026 *$ \\
\hline \multirow[t]{4}{*}{ Corpus callosum (body) } & $\alpha$ & 0.37 & $0.009 * *$ \\
\hline & $\beta$ & -0.94 & $<0.001 * * *$ \\
\hline & $\gamma$ & -0.99 & $<0.001 * * *$ \\
\hline & $\delta$ & -0.99 & $<0.001 * * *$ \\
\hline \multirow[t]{4}{*}{ Corpus callosum (genu) } & $\alpha$ & 0.17 & 0.240 \\
\hline & $\beta$ & -0.73 & $<0.001 * * *$ \\
\hline & $\gamma$ & -0.43 & $0.002 * *$ \\
\hline & $\delta$ & -0.74 & $<0.001 * * *$ \\
\hline \multirow[t]{4}{*}{ Corpus callosum (splenium) } & $\alpha$ & 0.52 & $0.001 * * *$ \\
\hline & $\beta$ & -0.85 & $<0.001 * * *$ \\
\hline & $\gamma$ & -0.99 & $<0.001 * * *$ \\
\hline & $\delta$ & -0.99 & $<0.001 * * *$ \\
\hline
\end{tabular}

The four Gompertz curve parameters indicate the initial onset of growth $(\beta)$ initial growth rate $(\gamma)$, a second reflection where growth begins to slow considerably $(\alpha)$, and a final linear growth rate $(\delta) ; * p<0.05, * * p<0.01, * * * p$ $<0.001$. 
Table 6. Statistical table for observational cohort analyses

Cross-sectional group difference in ELC (12-24 months of age) Association between MWF and SM content (12-24 months) Association between SM content and VDQ development Association between SM content and temporal WM $\beta$ Association between SM content and temporal WM $\gamma$ Association between SM content and parietal WM $\beta$ Association between SM content and parietal WM $\gamma$ Association between SM content and parietal WM $\delta$ Association between SM content and occipital WM $\beta$ Association between SM content and cerebellar WM $\alpha$ Association between SM content and cerebellar WM $\beta$ Association between SM content and cerebellar WM $\gamma$ Association between SM content and CC body $\beta$ Association between SM content and CC body $\gamma$ Association between SM content and CC body $\delta$ Association between SM content and CC genu $\beta$ Association between SM content and CC genu $\delta$ Association between SM content and CC splenium $\beta$ Association between SM content and CC splenium $\gamma$ Association between SM content and CC splenium $\delta$

\section{Data structure}

Normal distribution

Normal distribution

Normal distribution

Normal distribution

Normal distribution

Normal distribution

Normal distribution

Normal distribution

Normal distribution

Normal distribution

Normal distribution

Normal distribution

Normal distribution

Normal distribution

Normal distribution

Normal distribution

Normal distribution

Normal distribution

Normal distribution

Normal distribution

\section{Type of test \\ $t$ test}

Spearman's rank-order

Pearson's $r$

Pearson's $r$

Pearson's $r$

Pearson's $r$

Pearson's $r$

Pearson's $r$

Pearson's $r$

Pearson's $r$

Pearson's $r$

Pearson's $r$

Pearson's $r$

Pearson's $r$

Pearson's $r$

Pearson's $r$

Pearson's $r$

Pearson's $r$

Pearson's $r$

Pearson's $r$ $\begin{aligned} & \text { Power } \\ & 0.72 \\ & 0.56 \\ & 0.5 \\ & ">>0.90 \\ & ">>0.90 \\ & ">>0.90 \\ & ">>0.90 \\ & ">>0.90 \\ & ">>0.90 \\ & ">>0.90 \\ & ">>0.90 \\ & ">>0.90 \\ & ">>0.90 \\ & ">>0.90 \\ & ">>0.90 \\ & ">>0.90 \\ & ">>0.90 \\ & ">>0.90\end{aligned}$ impact on total cell number $(p>0.1$; Fig. $3 A$ ). Cell differentiation (O4+ cells) was significantly increased by 0.5 $\mu \mathrm{M}$ SM at 4 DIV (effect size 28.8\%; $p=0.0133$; Fig. 3B). SM at higher doses ( 5 and $25 \mu \mathrm{M})$ had no impact on cell differentiation ( $p>0.1$; Fig. $3 B$ ). 0.5 and $5 \mu \mathrm{M} \mathrm{SM}$ showed no impact on cell maturation (MBP+ cells, $p>0.1$; Fig. $3 C) ; 25 \mu \mathrm{M}$ SM resulted in a decrease of cell maturation, significantly at 4 and 7 DIV (effect size: $-23.6,-22.5 \% ; p$ $=0.0126$ and $p=0.0103$, respectively; Fig. $3 C$ ). Olesoxime treatment had no effect on total cell numbers, cell differentiation or maturation ( $p>0.1$; Fig. $3 A-C)$. Overall, these results show that $\mathrm{SM}$ treatment at $0.5 \mu \mathrm{M}$ resulted in increased OPC proliferation and differentiation (for representative images, see Fig. 4). With increased SM concentration, the effect gradually shifts toward a negative impact on differentiation and no impact on proliferation and maturation.

\section{Co-cultures}

We observed $0.5 \mu \mathrm{M}$ SM significantly increased axon myelination (co-localization of MBP and $\beta$-III-tubulin markers) at 16, 21, and 32 DIV (effect size: 16.8\%, 16.5\%, $64.9 \% ; p=0.0003, p=0.0002$, and $p=0.0001$, respectively; Fig. $3 D$, and see representative images in Fig. 5). On the contrary, SM treatment at higher doses significantly reduced myelination at 16,21 , and 32 DIV for $5 \mu \mathrm{M}$ (effect size: $-9.0 \%,-18.3 \%,-48.9 \% ; p=0.0047, p=$ 0.0006 , and $p=0.0015$, respectively; Fig. $3 D$ ) and for 25 $\mu \mathrm{M}$ (effect size: $-18.3 \%,-42.7 \%,-78.6 \% ; p<0.0095, p$ $<0.0001$, and $p<0.0001$, respectively; Fig. $3 D$ ). Treatment at $0.5 \mu \mathrm{M}$ SM significantly increased myelinating oligodendrocytes maturation (total MBP area) at 32 DIV (effect size: $53.3 \% ; p=0.0005$; Fig. $3 E$ ). Similarly to the effect observed on myelination, higher doses of SM resulted in a significant reduction of MBP signal at 16, 21, and $32 \mathrm{DIV}$; for $5 \mu \mathrm{M}$ (effect size: $-20.5 \%,-40.9 \%$, $-49.3 \% ; p=0.0084, p=0.0001$, and $p=0.0027$, respectively; Fig. $3 E$ ); and $25 \mu \mathrm{M}$ (effect size: $-28.0 \%$,
$-53.7 \%,-79.7 \% ; p=0.0163, p=0.0001$, and $p=$ 0.0001 , respectively; Fig. $3 E$ ); $0.5 \mu \mathrm{M}$ SM increased axonal growth ( $\beta$-III-tubulin) significantly at 21 DIV (effect size: $13.8 \%, p=0.0026$; Fig. $3 F$ ). Olesoxime significantly increased myelination at 16, 21, and 32 DIV (effect size: $15.1 \%, 28.7 \%, 22.2 \% ; p=0.0004, p=0.0001$, and $p=$ 0.0003 , respectively; Fig. $3 D)$ and axonal growth $(\beta-111-$ tubulin) at 21 DIV (effect size: $20.5 \% ; p=0.0008$; Fig. $4 F$;) and increased significantly myelinating oligodendrocyte (total MBP signal) at 32 DIV (effect size: $41.2 \%, p=$ 0.0019 ; Fig. $3 D$ ). These results suggest that $S M$ at concentration similar to that found in human CSF is capable of enhancing axon myelination and to some extent axonal growth in an in vitro coculture model of myelination (for representative images, see Fig. 5). With increased SM concentration, the effect gradually shifts toward a negative impact on myelination.

\section{Discussion}

To our knowledge, this is the first study in term-born neurotypically developing children that links dietary SM intake from infant nutrition products to cognitive development and brain myelination. Although preliminary, those findings provide important knowledge building and should be followed by efficacy studies. Our human observational data suggests that SM levels in infant nutrition products $(28-71 \mathrm{mg} / \mathrm{l})$ are positively correlated with changes in myelination in specific brain regions in the first two years of life. In addition, SM levels were also positively correlated with cognitive performance within normal ranges of development (ELC range $=91.5-104.2$ ). We found verbal functioning (e.g., auditory comprehension and memory, speaking ability and language formation; Dumont et al., 2000) to be more sensitive to early life nutrition than non-verbal functioning (e.g., visual processing and visual memory, motor control and coordination skills; Dumont et al., 2000). 
Table 7. Statistical table for in vitro analyses

\begin{tabular}{|c|c|c|c|c|c|}
\hline & & Data structure & Type of test & $95 \%$ co & cee interval \\
\hline & & & & $\mathrm{Cl}-2.5 \%$ & $\mathrm{Cl}-97.5 \%$ \\
\hline SM 0.5 & $\beta$-III-tubulin 2 & Normal after standardization & $t$ test on standardized data & -1.4 & 21.0 \\
\hline SM 0.5 & colocalization 1 & Normal after standardization & $t$ test on standardized data & -29.9 & -6.8 \\
\hline SM 0.5 & colocalization 2 & Normal after standardization & $t$ test on standardized data & -49.2 & -36.1 \\
\hline SM 0.5 & colocalization 3 & Normal after standardization & $t$ test on standardized data & -86.5 & -70.6 \\
\hline SM 0.5 & MBP1 & Normal after standardization & $t$ test on standardized data & -48.3 & -7.8 \\
\hline SM 0.5 & Myelination 1 & Normal after standardization & $t$ test on standardized data & -29.9 & -6.8 \\
\hline SM 0.5 & Myelination2 & Normal after standardization & $t$ test on standardized data & -49.2 & -36.1 \\
\hline SM 0.5 & Myelination3 & Normal after standardization & $t$ test on standardized data & -86.5 & -70.6 \\
\hline SM 0.5 & DAPI1 & Normal after standardization & $t$ test on standardized data & -11.9 & 17.8 \\
\hline SM 0.5 & DAPI2 & Normal after standardization & $t$ test on standardized data & -26.3 & 11.4 \\
\hline SM 0.5 & DAPI3 & Normal after standardization & $t$ test on standardized data & -16.2 & 13.7 \\
\hline SM 0.5 & MBP2 & Normal after standardization & $t$ test on standardized data & -37.4 & -7.5 \\
\hline SM 0.5 & MBP3 & Normal after standardization & $t$ test on standardized data & -26.6 & 5.4 \\
\hline SM 5 & $\beta$-III-tubulin 1 & Normal after standardization & $t$ test on standardized data & -3.5 & 8.3 \\
\hline SM 5 & $\beta$-III-tubulin 2 & Normal after standardization & $t$ test on standardized data & -17.2 & 18.0 \\
\hline SM 5 & $\beta$-III-tubulin 3 & Normal after standardization & $t$ test on standardized data & -11.5 & 8.2 \\
\hline SM 5 & colocalization 1 & Normal after standardization & $t$ test on standardized data & -13.7 & -4.2 \\
\hline SM 5 & colocalization 2 & Normal after standardization & $t$ test on standardized data & -24.5 & -12.2 \\
\hline SM 5 & colocalization 3 & Normal after standardization & $t$ test on standardized data & -69.0 & -28.9 \\
\hline SM 5 & MBP1 & Normal after standardization & $t$ test on standardized data & -33.1 & -8.0 \\
\hline SM 5 & MBP2 & Normal after standardization & $t$ test on standardized data & -49.8 & -32.0 \\
\hline SM 5 & MBP3 & Normal after standardization & $t$ test on standardized data & -72.4 & -26.2 \\
\hline SM 5 & Myelination 1 & Normal after standardization & $t$ test on standardized data & -13.7 & -4.2 \\
\hline SM 5 & Myelination2 & Normal after standardization & $t$ test on standardized data & -24.5 & -12.2 \\
\hline SM 5 & MBP2 & Normal after standardization & $t$ test on standardized data & -49.4 & 27.3 \\
\hline SM 5 & MBP3 & Normal after standardization & $t$ test on standardized data & -20.2 & 5.8 \\
\hline SM 25 & $\beta$-III-tubulin 1 & Normal after standardization & $t$ test on standardized data & -3.6 & 27.6 \\
\hline SM 25 & $\beta$-III-tubulin 2 & Normal after standardization & $t$ test on standardized data & 7.4 & 20.2 \\
\hline SM 25 & $\beta$-III-tubulin 3 & Normal after standardization & $t$ test on standardized data & -19.9 & 9.0 \\
\hline SM 25 & colocalization 1 & Normal after standardization & $t$ test on standardized data & 11.8 & 21.8 \\
\hline SM 25 & colocalization 2 & Normal after standardization & $t$ test on standardized data & 12.2 & 20.9 \\
\hline SM 25 & colocalization 3 & Normal after standardization & $t$ test on standardized data & 56.7 & 73.1 \\
\hline SM 25 & MBP1 & Normal after standardization & $t$ test on standardized data & -0.1 & 20.2 \\
\hline SM 25 & MBP2 & Normal after standardization & $t$ test on standardized data & -13.2 & 5.7 \\
\hline SM 25 & MBP3 & Normal after standardization & $t$ test on standardized data & 36.0 & 70.6 \\
\hline SM 25 & Myelination 1 & Normal after standardization & $t$ test on standardized data & 11.8 & 21.8 \\
\hline SM 25 & Myelination2 & Normal after standardization & $t$ test on standardized data & 12.2 & 20.9 \\
\hline SM 25 & Myelination3 & Normal after standardization & $t$ test on standardized data & 56.7 & 73.1 \\
\hline SM 25 & DAPI1 & Normal after standardization & $t$ test on standardized data & 9.1 & 48.6 \\
\hline SM 25 & DAPI2 & Normal after standardization & $t$ test on standardized data & -23.7 & 31.4 \\
\hline SM 25 & DAPI3 & Normal after standardization & $t$ test on standardized data & 0.8 & 36.4 \\
\hline SM 25 & O4-1 & Normal after standardization & $t$ test on standardized data & 9.7 & 49.4 \\
\hline SM 25 & O4-2 & Normal after standardization & $t$ test on standardized data & -23.3 & 31.4 \\
\hline SM 25 & $04-3$ & Normal after standardization & $t$ test on standardized data & 1.7 & 36.6 \\
\hline SM 25 & MBP1 & Normal after standardization & $t$ test on standardized data & 0.4 & 36.5 \\
\hline SM 25 & MBP2 & Normal after standardization & $t$ test on standardized data & -39.3 & 24.4 \\
\hline SM 25 & MBP3 & Normal after standardization & $t$ test on standardized data & -16.9 & 62.0 \\
\hline
\end{tabular}

$\mathrm{O} 4$ = olesoxime 


\subsection{6}

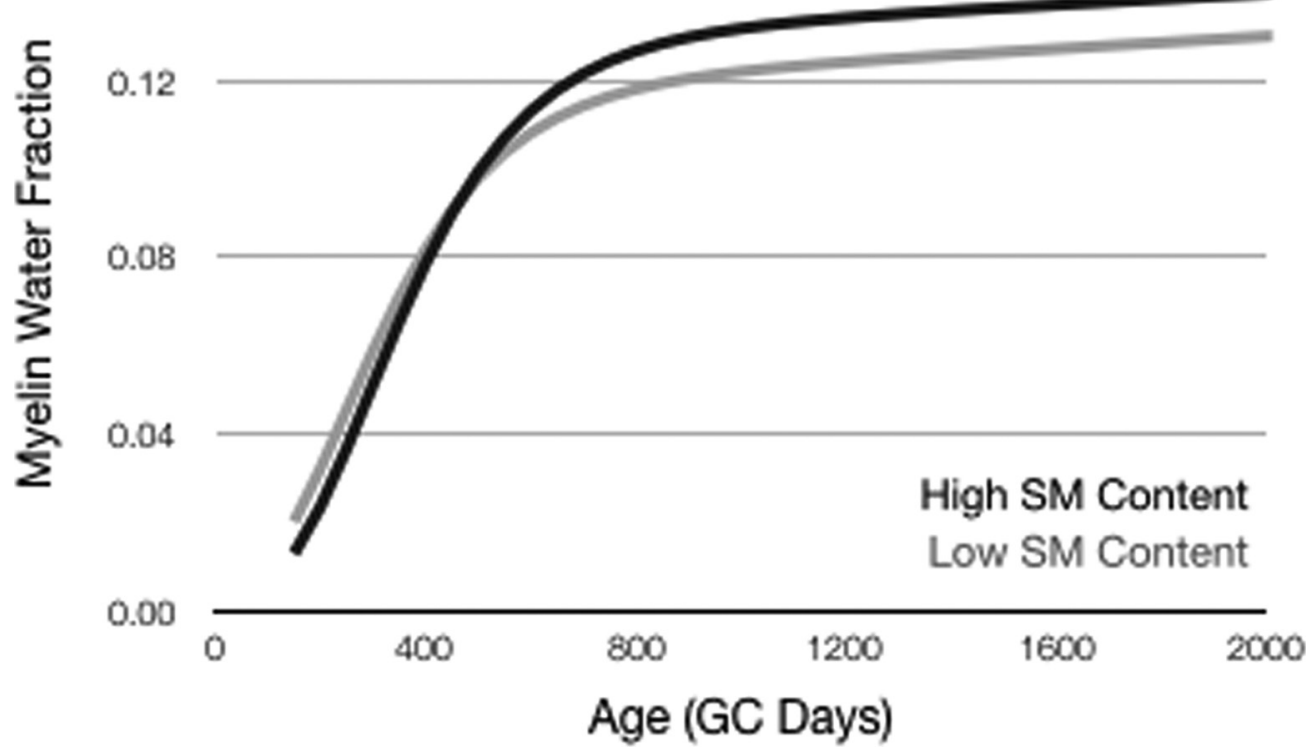

Figure 2. Mean whole-brain WM myelination trajectories derived from children who either received a product composition with high SM content $(71 \mathrm{mg} / \mathrm{l})$ or a lower SM content $(28 \mathrm{mg} / \mathrm{l})$ composition.

A

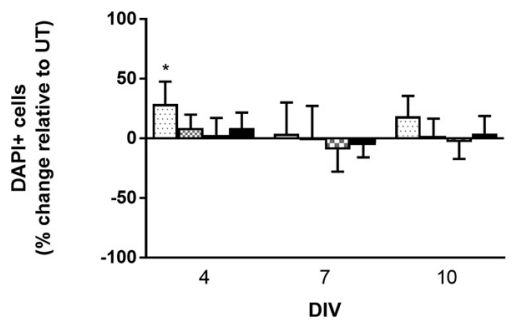

D

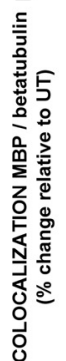

B

OL MYELINATION

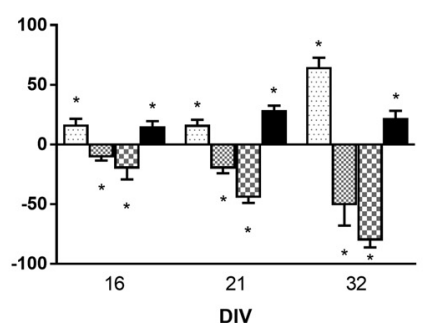

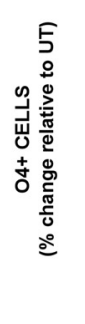

E

OPC DIFFERENTIATION

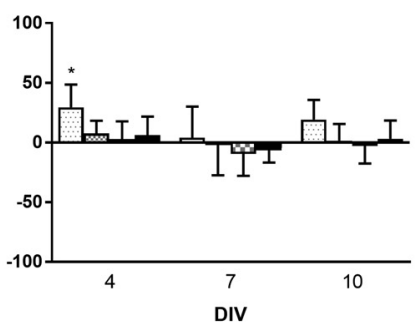

OL MATURATION

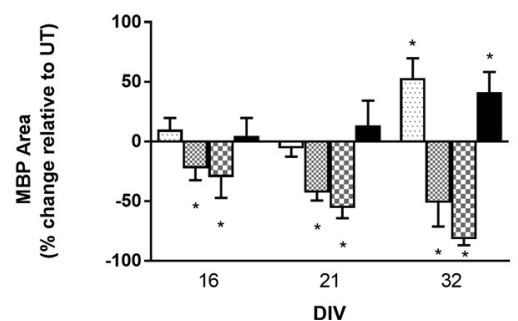

C

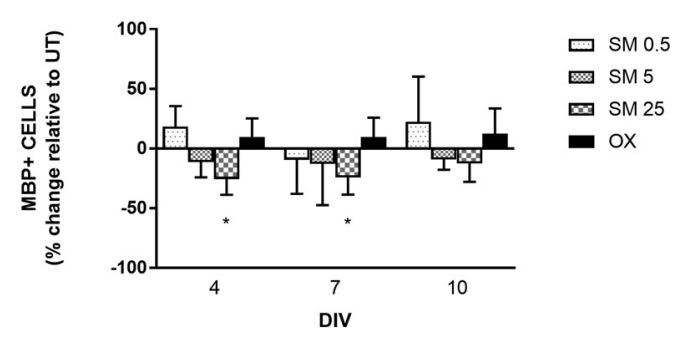

$\mathbf{F}$

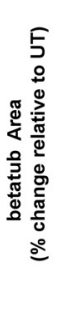

NEURITE NETWORK

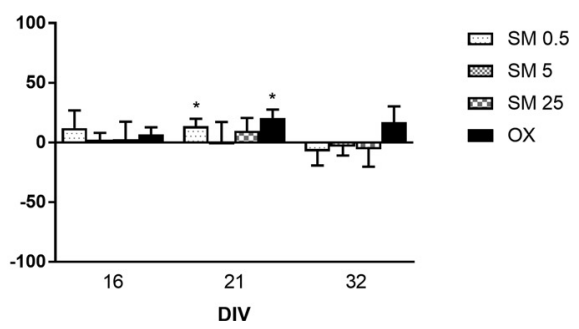

Figure 3. $\boldsymbol{A}-\boldsymbol{F}$, Impact of SM treatment on OPC proliferation, oligodendrocyte maturation, and differentiation in the pure OPC culture and on axon myelination in neuron-oligodendrocyte coculture per treatment group per DIV. Data presented are expressed as percentage change relative to untreated group and represent mean \pm SD for 4, 7, and 10 DIV (A-C) and for 16, 21, and 32 DIV (D-F). Treatment groups are untreated (UT), $0.5 \mu \mathrm{M}$ SM (SM 0.5), $5 \mu \mathrm{M}$ SM (SM 5), $25 \mu \mathrm{M}$ SM (SM 25), and $0.3 \mu \mathrm{M}$ olesoxime (OX; positive control), $N=3$ average of minimum two replicates per treatment group per time point. $\boldsymbol{A}$, OPC proliferation evaluated by total number of cells in percentage change relative to UT. $\boldsymbol{B}$, Oligodendrocyte differentiation evaluated by total number of O4+ cells in percentage change relative to UT. $\boldsymbol{C}$, Oligodendrocyte maturation evaluated by total number of MBP + cells in percentage change relative to UT. $\boldsymbol{D}$, Myelination in neuron-oligodendrocyte coculture evaluated by total colocalization of MBP and $\beta$-III-tubulin signal in percentage change relative to UT. $\boldsymbol{E}, \mathrm{MBP}$ immunofluorescence area in percentage change relative to UT. $\boldsymbol{F}, \beta$-III-tubulin immunofluorescence area in percentage change relative to UT. Asterisks indicates a significant difference from UT $(p<0.022$, applying the BenjaminiHochberg procedure to control the false positive discovery rate at $5 \%$ (on 84 performed tests), the threshold for significance was set at $p<0.022$. 


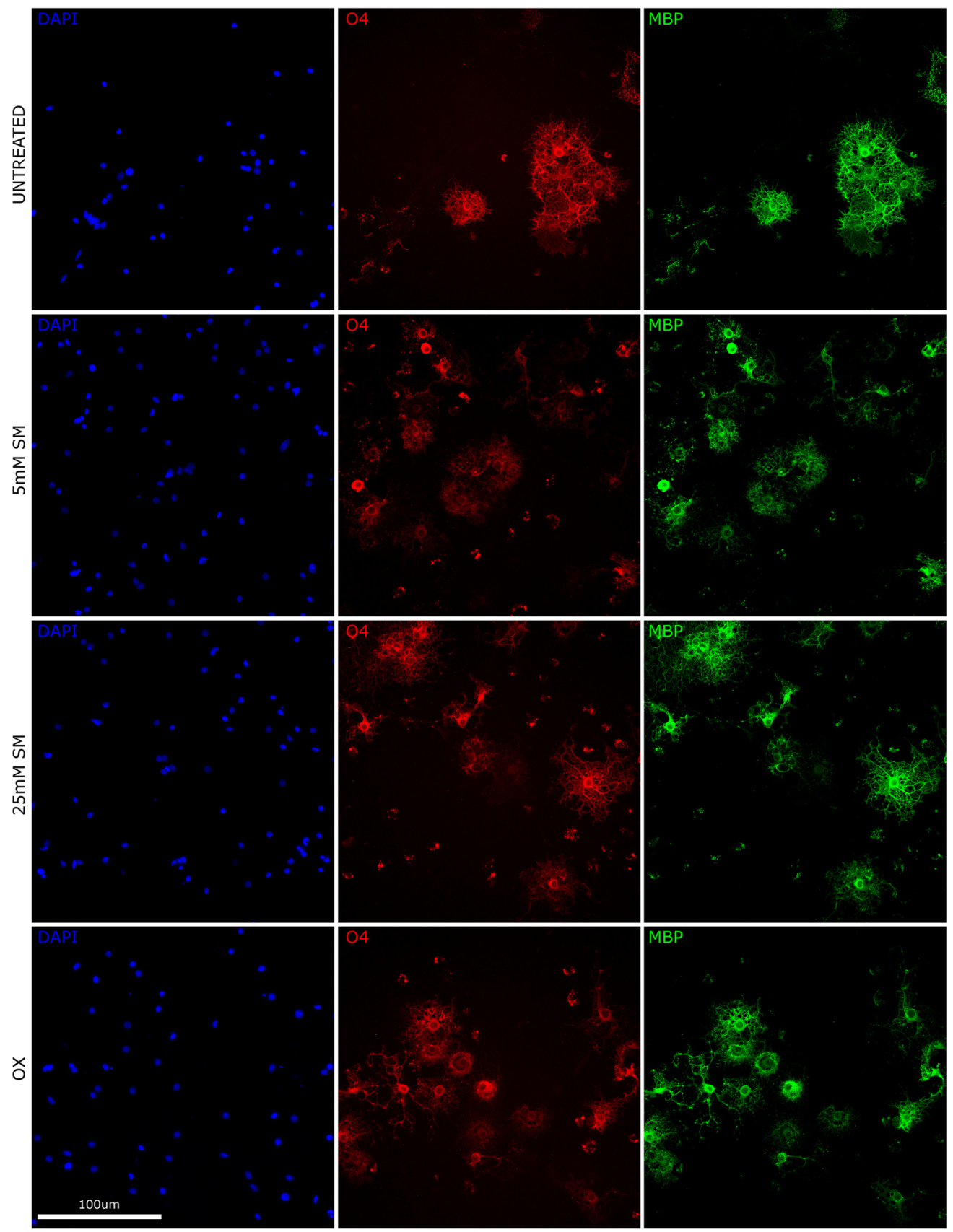

Figure 4. Representative illustration of OPC proliferation, oligodendrocyte maturation, and differentiation in the pure OPC culture per treatment groups at 10 DIV. Co-immunostaining with DAPI (blue), anti-O4 (red), and anti-MBP (green) of coculture at 21 DIV for the untreated (UT), $0.5 \mu \mathrm{M}$ SM (SM 0.5), $5 \mu \mathrm{M}$ SM (SM 5), $25 \mu \mathrm{M}$ SM (SM 25), and $0.3 \mu \mathrm{M}$ olesoxime (OX; positive control) groups. Proliferation was measured by number of cells stained with DAPI, maturation by number of $\mathrm{O} 4+$ cells, and differentiation by number of $\mathrm{MBP}+$ cells.

These association findings in typically developing children may complement the preliminary findings of Tanaka et al. (2013) in low birth weight infants. In this study, the authors reported an improvement of cognitive and behavioral outcomes at 12 and/or 18 months of age following a SM-fortified infant formula intervention in the first two months of life. Infants in that study were mostly mixed-fed (formula in addition to breast milk), thus effects cannot be attributed to the SM-fortified infant formula alone. Although exploratory, both studies may support the role of dietary SM in cognitive development from 12-24 months of age. Before any impact on cognitive development, our findings suggest a modulation of myelin development in the brain, possibly via the involvement of SM in the structure and function of myelin (Ledesma et al., 1999; Don et al., 2014). Higher levels of SM in the first three months of life were associated ( $p<0.05$ FDR) with higher levels of brain myelin content in the second year of life, particularly in the cerebellum, occipital lobe, visual cortex, internal capsule, parietal lobe, and motor cortices. Furthermore, 


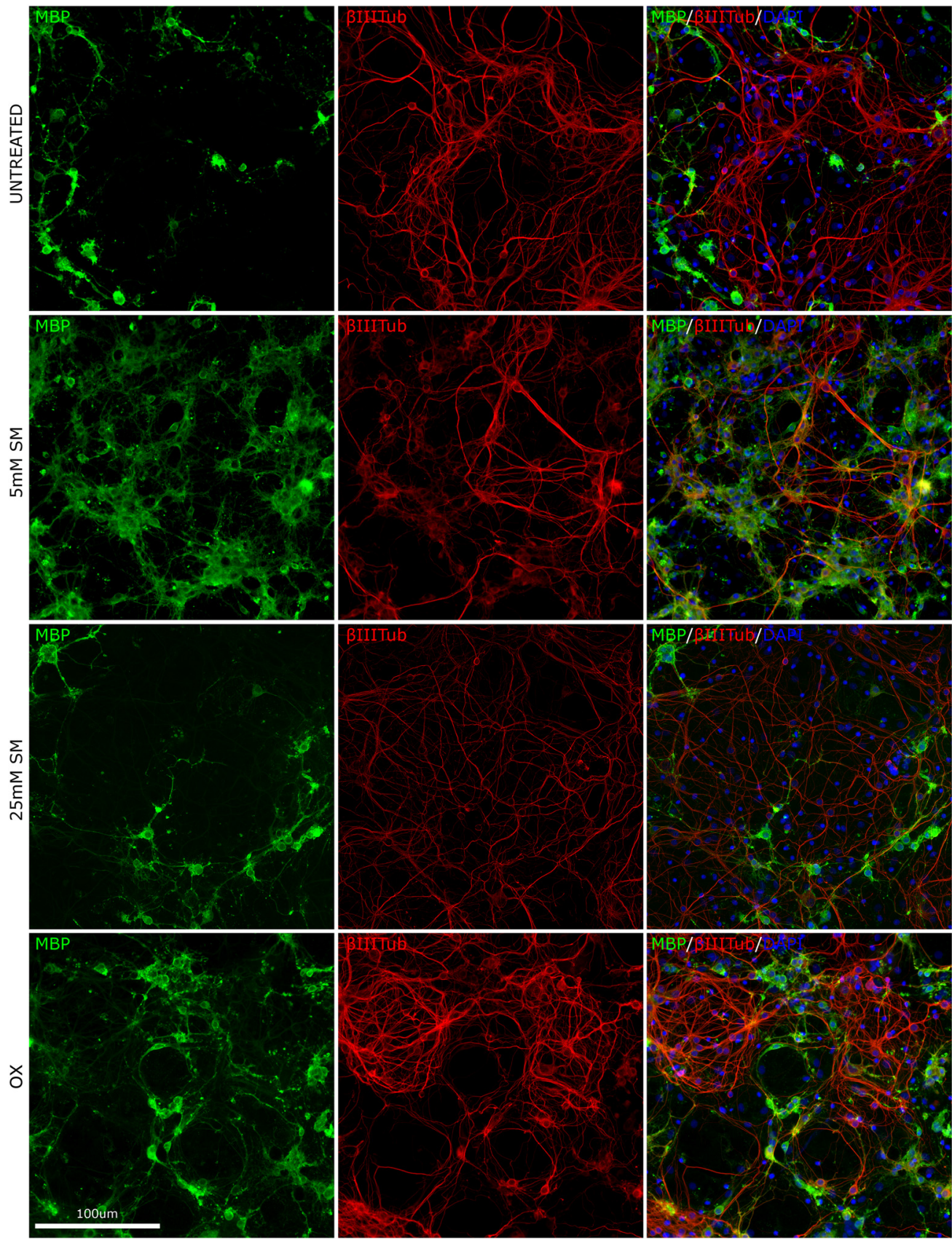

Figure 5. Representative illustration of neuron-oligodendrocyte coculture at 32 DIV. Coimmunostaining with MPB (green), anti- $\beta$-IIItubulin (red), and DAPI (blue) of coculture at 32 DIV for the untreated (UT), $0.5 \mu$ M SM (SM 0.5), $5 \mu$ M SM (SM 5), $25 \mu$ M SM (SM 25), and $0.3 \mu \mathrm{M}$ olesoxime (OX; positive control) groups. Myelination was quantified by the overlap of MBP (green) and $\beta$-III-tubulin (red) staining.

our findings indicate an impact of SM on the pattern of developmental myelination in favor of a later onset as well as a more prolonged rate of myelination. This seems particularly relevant in the context of development, as dysregulations and deviations from the developmental pattern, rather than overall myelin quantity, may contribute to the production of human psychiatric disturbances, such as autism spectrum disorders, learning deficits, sensory processing delay disorder, attention deficit hyperactivity disorder, and Retts syndrome (Herbert et al., 2004; Bartzokis 2005; Bercury and Macklin, 2015).
Our supporting in vitro studies show that physiologic levels of SM positively affect the proliferation, differentiation and myelination capacity of oligodendrocyte while higher concentrations may have a negative impact. However, it is unlikely that SM itself is transported to the brain and incorporated into myelin sheath directly. SM is metabolized in the small intestine into ceramide and sphingosine by alkaline sphingomyelinase (SMase; Nyberg et al., 1997; Ohlsson et al., 2010). Additional studies show that sphingosine and ceramide derived from sphingolipid hydrolysis are rapidly taken up by intestinal cells, then 
either degraded into fatty acids or reincorporated into complex sphingolipids that could be associated with lipoprotein particles including chylomicron, very low-density lipoprotein (VLDL), LDL, and high-density lipoprotein (HDL; Nilsson and Duan, 2006). SM has been shown to be the major sphingolipid in LDL and HDL (Vesper et al., 1999). Injection of HDL containing radiolabeled SM suggest some direct uptake of SM to the brain, however the majority was resynthesized in the brain (Bentejac et al., $1988,1989)$. Since the brain appears to be capable of de novo synthesis of sphingolipids, SM and its constituents could be used for de novo synthesis of main myelin lipids such as cerebrosides to support myelin formation during brain development. However, the time course of intact de novo synthesis of sphingolipids in newborns is not yet well understood. It is likely that infants depend on external sources in their first weeks of life. This hypothesis is supported by the greater uptake of radiolabeled SM injected in blood observed in 20-d-old rats compare to adult rats (Bentejac et al., 1989). Additional experiments are necessary to fully characterize how orally ingested SM contribute to myelinogenesis, especially at different stages of infant and child development.

In our in vitro experiments, we used SM in a liposome form to facilitate its cellular uptake. We observed that the lowest concentration of SM, corresponding to the one found in human CSF, promotes OPC proliferation and differentiation in pure OPC cultures and enhances myelination in coculture. Higher concentrations of SM have no effect on OPC proliferation and OPC maturation or negatively affect OPC differentiation, OL myelination, and OL maturation. While the low SM concentrations transiently enhanced dendritic arborization in coculture, higher concentrations failed to yield an effect. Our results suggest that SM may have different effects on oligodendrocyte and neuron physiology that may be linked to its role in multiple cell processes. Additional experiments will be needed to characterize the exact mechanism of SM in myelination and axonal growth.

Limitations of our study include the retrospective analyses of the infant nutrition products. While product and feeding information was acquired during the study period, product samples were acquired at the study end and SM analyses were performed retrospectively using a single time assay. Thus, the nutritional formulations of the reported products may have changed in the years from the earliest imaging data up to the time of our nutritional analysis. Furthermore, we observed a temporal delay between infant feeding and impact on myelination and cognitive development. It is likely that follow-on complementary feeding and nutrition, as well as other environmental influences previously associated with cognitive development and outcomes in children, such as parentchild interaction (Swain et al., 2007), physical activity levels (Best, 2010), and sleep duration and quality (Peirano and Algarín, 2007) are unexamined contributors to our results. Another important caveat to our study is the absence of bioavailability information in both our observational as well as our in vitro data. As discussed above, $\mathrm{SM}$ is metabolized and resynthesized and relevant amounts are unlikely to reach the brain directly (Nyberg et al., 1997). Observed associations may therefore be the result of SM metabolites influencing brain and cognitive development rather than SM itself. Indeed, more studies are needed to understand bioavailability, brain uptake and mechanisms of action of dietary SM and its metabolites. The relatively small sample size per sub-group of infant nutrition product-fed infants $(N=21, N=28$, and $N=39$, respectively) should be considered when interpreting the findings of the study. Although not unusual for pediatric neuroimaging studies, the sample sizes are low. At this stage, we consider our findings preliminary and in support of the need to further investigate the role of dietary SM in normal brain and cognitive development.

In conclusion, our first findings from a clinical observational study indicate an impact of dietary SM on cognitive development in healthy term-born children. This effect may be mediated through oligodendrocytes proliferation and differentiation as supported by our in vitro experiments. Randomized controlled trials, ideally of longitudinal nature to best reflect development and maturation, are needed to substantiate efficacy for dietary SM related cognitive benefits. In addition, complementary preclinical studies on bioavailability and brain uptake of SM and its metabolites are needed. Furthermore, SM doses for oral intake and efficacy need to be identified to provide appropriate recommendations for infant and child nutrition.

\section{References}

Assi E, Cazzato D, De Palma C, Perrotta C, Clementi E, Cervia D (2013) Sphingolipids and brain resident macrophages in neuroinflammation: an emerging aspect of nervous system pathology. Clin Dev Immunol 2013:309302.

Avants BB, Tustison NJ, Song G, Cook PA, Klein A, Gee JC (2011) A reproducible evaluation of ANTs similarity metric performance in brain image registration. Neuroimage 54:2033-2044.

Babin F, Sarda P, Limasset B, Descomps B, Rieu D, Mendy F, Crastes de Paulet A (1993) Nervonic acid in red blood cell sphingomyelin in premature infants: an index of myelin maturation? Lipids 28:627-630.

Bartzokis G (2005) Brain myelination in prevalent neuropsychiatric developmental disorders: primary and comorbid addiction. Adolesc Psychiatry 29:55-96.

Benjamini Y, Hochberg Y (1995) Controlling the false discovery rate: a practical and powerful approach to multiple testing. J R Stat Soc Series B Stat Methodol 57:289-300.

Bentejac M, Lecerf J, Bugaut M, Delachambre MC (1988) Turnover and uptake of double-labelled high-density lipoprotein sphingomyelin in the adult rat. Biochim Biophys Acta 959:349-360.

Bentejac M, Bugaut M, Delachambre MC, Lecerf J (1989) Utilization of high-density lipoprotein sphingomyelin by the developing and mature brain in the rat. J Neurochem 52:1495-1500.

Bercury KK, Macklin WB (2015) Dynamics and mechanisms of CNS myelination. Dev Cell 32:447-458.

Best JR (2010) Effects of physical activity on children's executive function: contributions of experimental research on aerobic exercise. Dev Rev 30:331-551.

Bitman J, Wood DL, Mehta NR, Hamosh P, Hamosh M (1984) Comparison of the phospholipid composition of breast milk from mothers of term and preterm infants during lactation. Am J Clin Nutr 40:1103-1119.

Cilla A, Diego Quintaes K, Barberá R, Alegría A (2016) Phospholipids in human milk and infant formulas: benefits and needs for correct infant nutrition. Crit Rev Food Sci Nutr 56:1880-1892. 
Dean DC 3rd, Dirks H, O'Muircheartaigh J, Walker L, Jerskey BA, Lehman K, Han M, Waskiewicz N, Deoni SC (2014a) Pediatric neuroimaging using magnetic resonance imaging during nonsedated sleep. Pediatr Radiol 44:64-72.

Dean DC 3rd, O'Muircheartaigh J, Dirks H, Waskiewicz N, Lehman K, Walker L, Han M, Deoni SC (2014b) Modeling healthy male white matter and myelin development: 3 through 60 months of age. Neuroimage 84:742-752.

Deoni SC (2011) Magnetic resonance relaxation and quantitative measurement in the brain. Methods Mol Biol 711:65-108.

Deoni SC, Kolind SH (2015) Investigating the stability of mcDESPOT myelin water fraction values derived using a stochastic region contraction approach. Magn Reson Med 73:161-169.

Deoni SC, Williams SC, Jezzard P, Suckling J, Murphy DG, Jones DK (2008) Standardized structural magnetic resonance imaging in multicentre studies using quantitative T1 and T2 imaging at $1.5 \mathrm{~T}$. Neuroimage 40:662-671.

Deoni SC, Dean DC 3rd, O'Muircheartaigh J, Dirks H, Jerskey BA (2012) Investigating white matter development in infancy and early childhood using myelin water faction and relaxation time mapping. Neuroimage 63:1038-1053.

Deoni SC, Dean DC 3rd, Walker L, Dirks H, O’Muircheartaigh J (2014) Nutritional influences on early white matter development: response to Anderson and Burggren. Neuroimage 100:703-705.

Don AS, Hsiao JH, Bleasel JM, Couttas TA, Halliday GM, Kim WS (2014) Altered lipid levels provide evidence for myelin dysfunction in multiple system atrophy. Acta Neuropathol Commun 2:150.

Dumont R, Cruse CL, Alfonso V, Levine C (2000) Book review: Mullen scales of early learning: AGS edition. J Psychoeduc Assess 18: 381-389.

Fields RD (2008) Oligodendrocytes changing the rules: action potentials in glia and oligodendrocytes controlling action potentials. Neuroscientist 14:540-543.

Gault CR, Obeid LM, Hannun YA (2010) An overview of sphingolipid metabolism: from synthesis to breakdown. Adv Exp Med Biol 688:1-23.

Giuffrida F, Cruz-Hernandez C, Flück B, Tavazzi I, Thakkar SK, Destaillats F, Braun M (2013) Quantification of phospholipids classes in human milk. Lipids 48:1051-1058.

Herbert MR, Ziegler DA, Makris N, Filipek PA, Kemper TL, Normandin JJ, Sanders HA, Kennedy DN, Caviness VS Jr (2004) Localization of white matter volume increase in autism and developmental language disorder. Ann Neurol 55:530-540.

Hirabayashi Y, Furuya S (2008) Roles of I-serine and sphingolipid synthesis in brain development and neuronal survival. Prog Lipid Res 47:188-203.

Ledesma MD, Brügger B, Bünning C, Wieland FT, Dotti CG (1999) Maturation of the axonal plasma membrane requires upregulation of sphingomyelin synthesis and formation of protein-lipid complexes. EMBO J 18:1761-1771.

Lingwood D, Simons K (2010) Lipid rafts as a membrane-organizing principle. Science 327:46-50.

Maceyka M, Spiegel S (2014) Sphingolipid metabolites in inflammatory disease. Nature 510:58-67.

Magalon K, Zimmer C, Cayre M, Khaldi J, Bourbon C, Robles I, Tardif G, Viola A, Pruss RM, Bordet T, Durbec P (2012) Olesoxime accelerates myelination and promotes repair in models of demyelination. Ann Neurol 71:213-226.

Mandal R, Guo AC, Chaudhary KK, Liu P, Yallou FS, Dong E, Aziat F, Wishart DS (2012) Multi-platform characterization of the human cerebrospinal fluid metabolome: a comprehensive and quantitative update. Genome Med 4:38.

Merrill AH Jr, Sandhoff K (2002) Sphingolipids: metabolism and cell signaling. In: Biochemistry of lipids, lipoproteins and membranes, Chap 14, pp 373-407. Amsterdam: Elsevier.

Mullen EM (1995) Mullen scales of early learning. Circle Pines, MN: American Guidance Services.

Nilsson A, Duan RD (2006) Absorption and lipoprotein transport of sphingomyelin. J Lipid Res 47:154-171.

Nyberg L, Nilsson Å, Lundgren P, Duan RD (1997) Localization and capacity of sphingomyelin digestion in the rat intestinal tract. $J$ Nutr Biochem 8:112-118.

O'Muircheartaigh J, Dean DC 3rd, Dirks H, Waskiewicz N, Lehman K, Jerskey BA, Deoni SC (2013) Interactions between white matter asymmetry and language during neurodevelopment. $\mathrm{J}$ Neurosci 33:16170-16177.

O'Muircheartaigh J, Dean DC, 3rd, Ginestet CE, Walker L, Waskiewicz N, Lehman K, Dirks H, Piryatinsky I, Deoni SC (2014) White matter development and early cognition in babies and toddlers. Hum Brain Mapp 35:4475-4487.

Ohlsson L, Hertervig E, Jönsson BA, Duan RD, Nyberg L, Svernlöv R, Nilsson A (2010) Sphingolipids in human ileostomy content after meals containing milk sphingomyelin. Am J Clin Nutr 91:672-678.

Oshida K, Shimizu T, Takase M, Tamura Y, Shimizu T, Yamashiro Y (2003) Effects of dietary sphingomyelin on central nervous system myelination in developing rats. Pediatr Res 53:589-593.

Peirano PD, Algarín CR (2007) Sleep in brain development. Biol Res 40:471-478.

Pierre M (1984) Myelin. New York: Springer.

Smith SM, Nichols TE (2009) Threshold-free cluster enhancement: addressing problems of smoothing, threshold dependence and localisation in cluster inference. Neuroimage 44:83-98.

Stiles J, Jernigan TL (2010) The basics of brain development. Neuropsychol Rev 20:327-348.

Swain JE, Lorberbaum JP, Kose S, Strathearn L (2007) Brain basis of early parent-infant interactions: psychology, physiology, and in vivo functional neuroimaging studies. J Child Psychol Psychiatry 48:262-287.

Tanaka K, Hosozawa M, Kudo N, Yoshikawa N, Hisata K, Shoji H, Shinohara K, Shimizu T (2013) The pilot study: sphingomyelinfortified milk has a positive association with the neurobehavioural development of very low birth weight infants during infancy, randomized control trial. Brain Dev 35:45-52.

Tau GZ, Peterson BS (2010) Normal development of brain circuits. Neuropsychopharmacology 35:147-168.

Timby N, Domellöf E, Hernell O, Lönnerdal B, Domellöf M (2014) Neurodevelopment, nutrition, and growth until 12 mo of age in infants fed a low-energy, low-protein formula supplemented with bovine milk fat globule membranes: a randomized controlled trial. Am J Clin Nutr 99:860-868.

Vesper H, Schmelz EM, Nikolova-Karakashian MN, Dillehay DL, Lynch DV, Merrill AH Jr (1999) Sphingolipids in food and the emerging importance of sphingolipids to nutrition. J Nutr 129: 1239-1250.

Vickers MH, Guan J, Gustavsson M, Krägeloh CU, Breier BH, Davison M, Fong B, Norris C, McJarrow P, Hodgkinson SC (2009) Supplementation with a mixture of complex lipids derived from milk to growing rats results in improvements in parameters related to growth and cognition. Nutr Res 29:426-435. 